\title{
Dynamic analysis of the turbo - generator foundation structure
}

\author{
Jan Benčat ${ }^{1,{ }^{*}}$, Michal Tomko ${ }^{2}$, and Michal Lukáć ${ }^{3}$ \\ ${ }^{1}$ University of Žilina; Institute of Competitiveness and Innovations, Žilina, Slovakia \\ ${ }^{2}$ TUKE Košice, Faculty of Civil Engineering, Košice, Slovakia \\ ${ }^{3}$ University of Žilina; Faculty of Mechanical Engineering, Žilina, Slovakia
}

\begin{abstract}
This paper provides view of current trends in the field of testing and numerical analysis of dynamic loading structures. It describes what is the role of structure dynamic characteristic analysis in the management of the structures construction and maintenance in power plant industry. The main objective of this study is the dynamic analysis of power plant turbogenerator foundation structure (TGFS) of electrical industry operation. Main purpose of performed study in 2017 was to check dynamic stiffness TGFS after fifty years TG (100 MW) performance which then enabled to prepare relevant data for making design renovation and strengthening of the TGSF.
\end{abstract}

\section{Introduction}

The dynamic analysis of TGFS of two parts: (i) analytical approach with initial numerical FEM analysis and (ii) experimental analysis of existing foundation structure via its experimental dynamic response due to vibration mechanical exciter and impulse apparatus LFWD (Light Falling Weight Device). Results of the experimental tests (ET) in situ then enabled to design of the existing foundation structure strengthening for the future TG performance and then update initial foundation structural model for final numerical analysis $[1,2]$. According to the TGFS experimental analysis results and numerical calculation results was created updated FEM foundation structural model for calculation of the predicting dynamic response of the strengthening foundation structure (FS). The objectives of the final numerical calculation results were to compare the calculated and experimental measured natural frequencies value check of resonance conditions and whether vibrations magnitude of renovated TGFS will satisfy of the relevant code limits e.g. ISO 1940-1 (1993) or the machine manufacturer prescriptions. After the finishing of TGFS strengthening works are planed experimental tests to determine of turbo-generator foundation structure real dynamic stiffness via comparison of measured real structure dynamic response parameters values to final numerical calculation results.

\footnotetext{
* Corresponding author: jan.bencat@gmail.com
} 


\section{Numerical analysis}

Before experimental analysis of TGFS-6 it was performed to start procedures for the FE modelling [3] of existing foundation structure TGFS-6 along with supporting soil and numerical dynamic analysis of it. For creating FE initial model (FEM1) were as an input data used mainly relevant project parameters. Dynamic numerical analysis was solved using ANSYS software package which provides an effective computational environment to perform these types of analyses. Calculation of the natural frequencies and corresponding modes of vibration forms basis for the determination of the dynamic parameters of TGFS. As an example of the comparison FEM1 calculation results to experimental results of the natural frequencies value $f(j)$ of the TGFS- 6 vibration are presented in Table 1 . The Fig. 1 shows an example of the FEM1 basic natural modes with frequencies calculation results.

The numerical determination of the natural frequencies and modes of TG foundation structure vibration was in this case fairly difficult and it was advised their verification by experimental measurements. Results of TGFS experimental analysis were fully utilized for creating final calculation model (FEM2) of the TGFS - 6 which also comprising of the designer strengthening elements and whole FS, Fig.2. The updated FEM2 [4] for future offers calculation for reconstructed TGFS-6, e.g.: (i) natural frequencies and corresponding modes of vibration (free vibration analysis, eigen analysis); (ii) dynamic amplitude calculation due to turbine operation and critical regimes to check that the same are within the acceptable relevant code limits or as prescriptions limits of the equipment supplier (forced vibration analysis); (iii) dynamic response due to seismic acting (e.g. seismic analysis) and (iv) dynamic stresses amplitude calculation to check the stresses induced in the different structural element like beams, columns and slabs (pseudo-static).

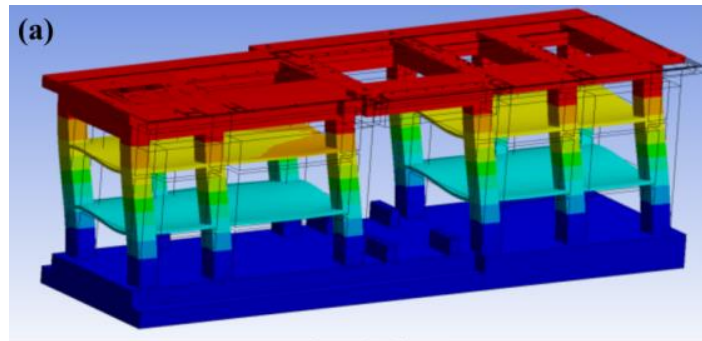

$f_{01}=4.42 \mathrm{~Hz}$

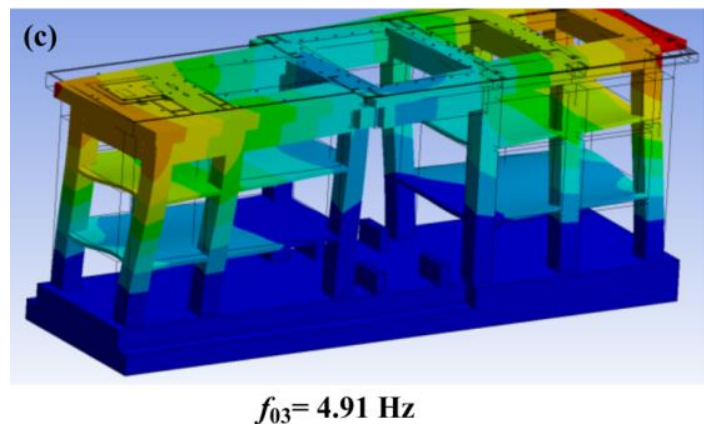

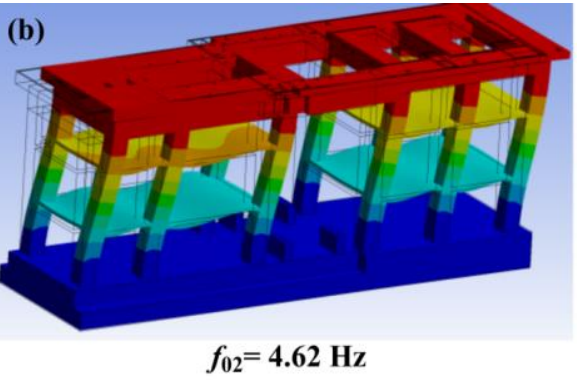

(d)

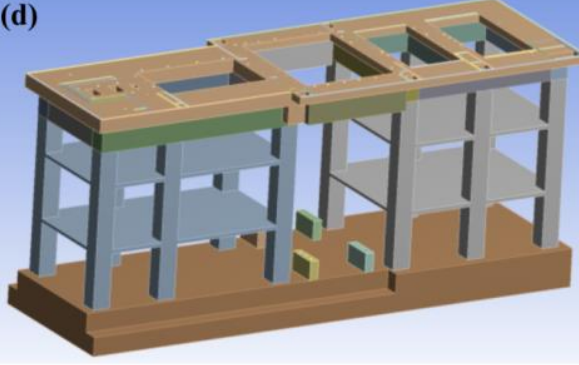

FEM 1

Fig. 1. Example of initial FEM analysis results: (a), (b), (c) - Basic modes of TGFS-6 vibration; (d) - FEM1 . 
Table1. Natural frequencies - numerical and experimental values comparison.

\begin{tabular}{|c|c|c|c|c|c|}
\hline \multicolumn{6}{|c|}{ TGFS - 6 Values of Basic Natural Frequencies $-f_{0 \mathrm{i}}(\mathrm{Hz})$} \\
\hline \multirow{2}{*}{$\begin{array}{c}\text { No. } \\
(i)\end{array}$} & \multicolumn{2}{|c|}{ Numerical } & \multicolumn{2}{|c|}{ Experimental } & \multirow{2}{*}{ Differences $/ \Delta$} \\
\hline & FEM1 & FEM2 & EXP1 & EXP2 $\left.^{* *}\right)$ & \\
\hline 1 & 4.42 & 4.10 & $\left.3.57^{*}\right)(3.40)$ & - & $\Delta=19.2 \%$ \\
\hline 2 & 4.62 & 6.14 & $4.30 *)(4.70)$ & - & $\Delta=7.0 \%$ \\
\hline 3 & 4.91 & 7.21 & Not evaluated & - & - \\
\hline 4 & 9.74 & 11.12 & $\mathbf{5 . 6 9 ^ { * } )}(6.99 ; 7.03)$ & - & $\Delta=41.8 \%$ \\
\hline 5 & 12.90 & 12.21 & 9.94 & - & $\Delta=33.36 \%$ \\
\hline
\end{tabular}

NOTE: $\quad \Delta \quad$ Natural frequencies numerical and experimental values differences

*) Natural frequencies experimental values determined by cross power spectral densities and proved by coherence and phase functions.

**) Frequency values will be available from test after repair of structure.

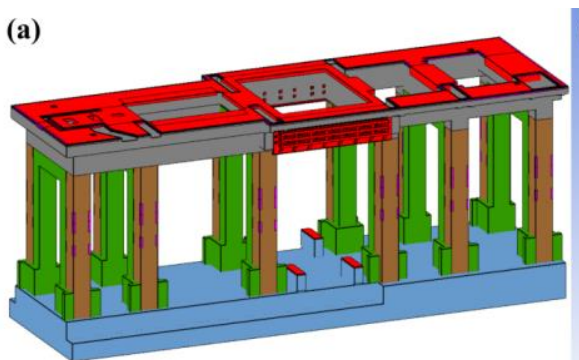

FEM 2

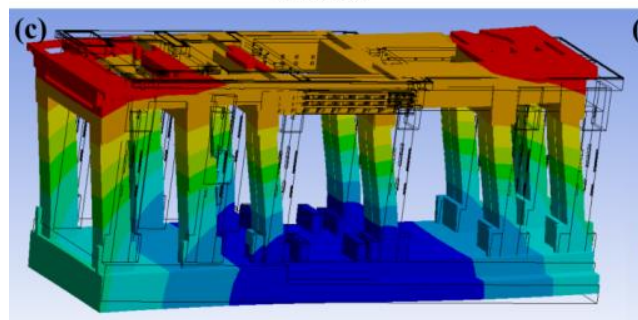

$f_{02}=6.14 \mathrm{~Hz}$

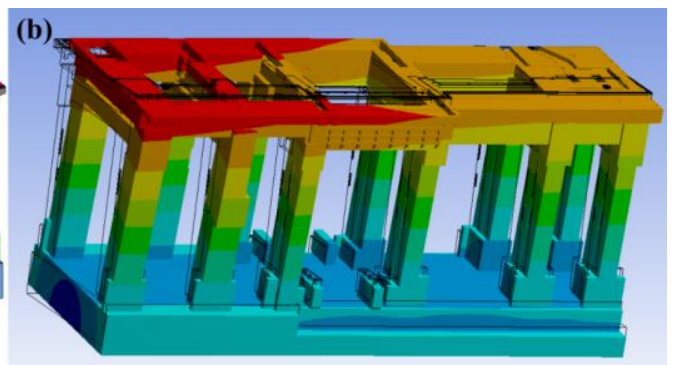

$f_{01}=4.10 \mathrm{~Hz}$

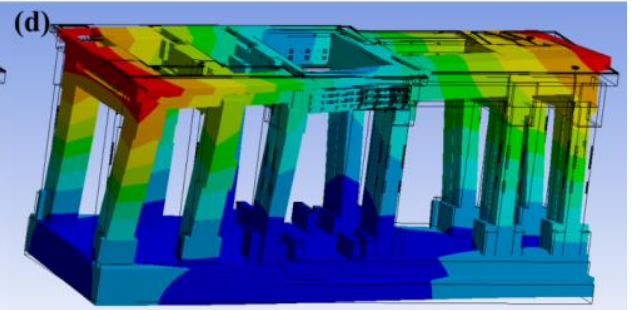

$f_{03}=7.21 \mathrm{~Hz}$

Fig. 2. Example of final FEM analysis results: (a) - FEM2; (b), (c), (d) - Basic modes of TGFS-6 vibration.

\section{Experimental analysis}

Before the performance of the final FEM2 numerical calculation of TGFS it was needed to perform experimental measurements of existing TGFS and its dynamic analysis. This was achieved via dynamic tests with harmonic and impulse forced vibration of foundation 
structure to determine the basic foundation dynamic parameters and then determine the natural frequencies and damping for achievable modes of vibration.

Experimental tests (ET). The mentioned TGFS foundation consists of an RCC top deck slab with supporting structure of beams and columns and a foundation rafts system resting on soil, Fig.3. From the top deck all the equipment including turbine, generator and other rotary equipment before experimental tests were removed. The experimental tests (EXP1 series) were carried out on the top deck slab and the raft of TGFS-6. The dimensions of the TGFS-6 are described in details in [1]. For the purpose of measuring forced vibration modes of the FS a spatial network of points has been chosen, Fig.3. In all ET points time history of structure acceleration amplitudes were measured in the three or two perpendicular directions. Harmonically variable forces were produced by vibration mechanical exciter (Fig.4) with the max force amplitude $0-11.5 \mathrm{kN}(\sim 10 \mathrm{~Hz})$ working in relevant positions (Fig.3) to achieve dynamic response of structure in one of excepted basic structure vibration mode. Impulse forces were produced by impulse apparatus (Light Falling Weight Device - LFWD). The twelve acceleration transducers of type KB $12 \mathrm{VD}$ $(1-4000 \mathrm{~Hz})$ were used for signal amplifiers and low-band pass filters and then recorded by portable notebook with relevant software coupled with needful hardware facilities in test measuring station.
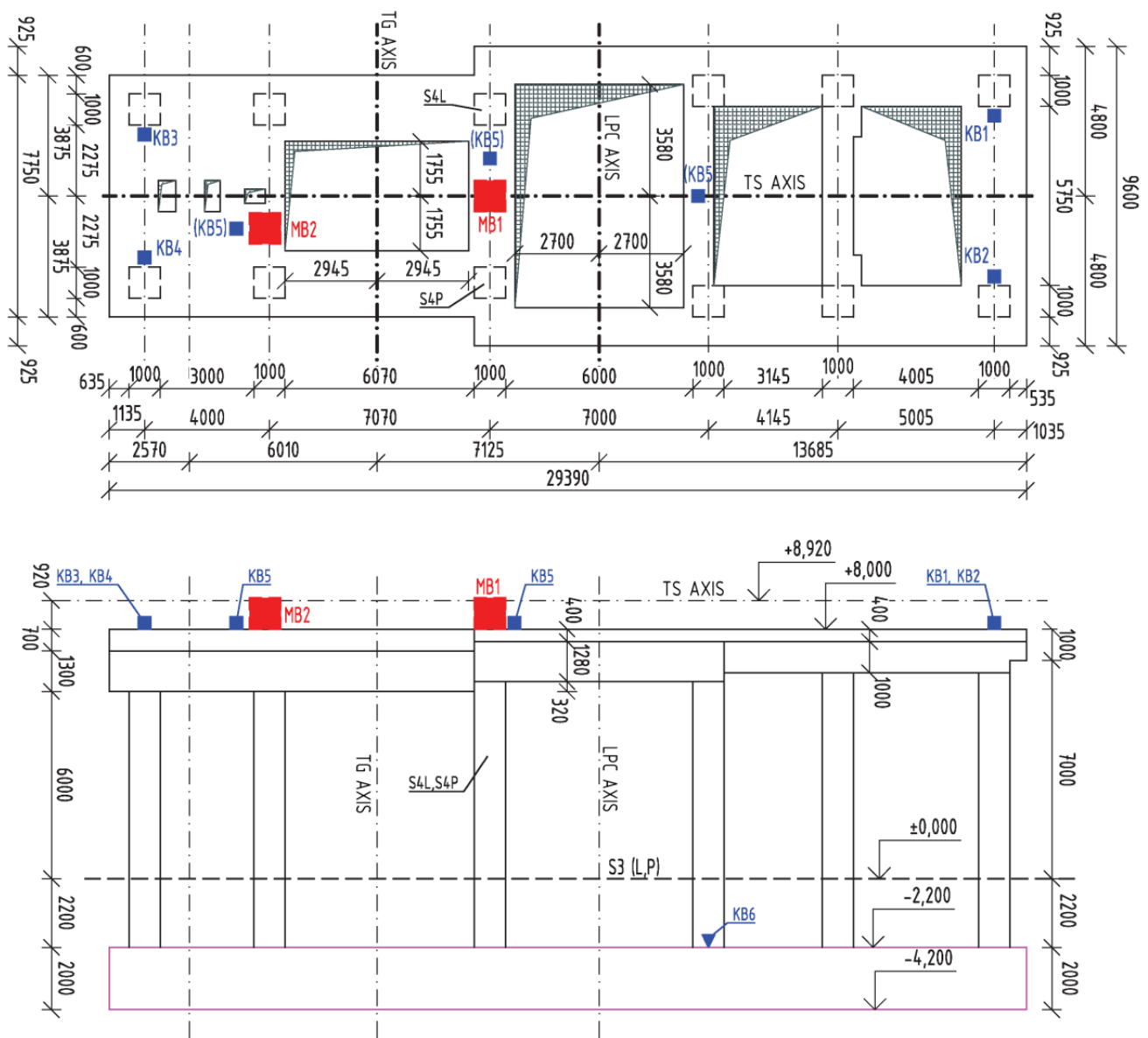

LEGEND: $\quad$ MB1,MB2 - mechanical exciter positions; (MB - ZU; 1-15,5 Hz)

$\mathrm{KB} 1 \ldots \mathrm{KB} 12$ - accelerometers position; (KB12VD;1-4000 Hz).

Fig. 3. TGFS with positions of measuring points and exciter positions layout. 
The signals from measurement devices (pick-ups) were transmitted to the recording technique in the measuring station (MS) by special low noise cables, Fig.5. The amplitude, correlation and spectral analysis of recorded signals were performed in laboratory UZ Zilina, see also [1].

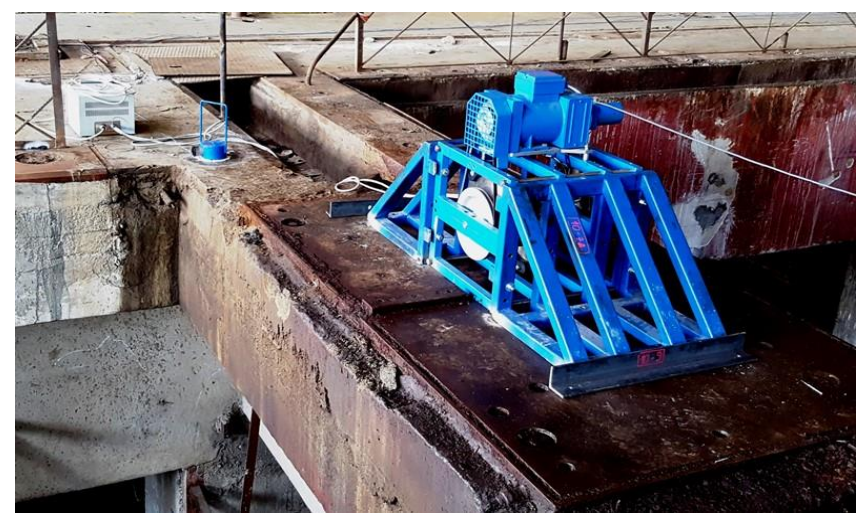

Fig. 4. Mechanical exciter at MB1 position on TGFS-6.

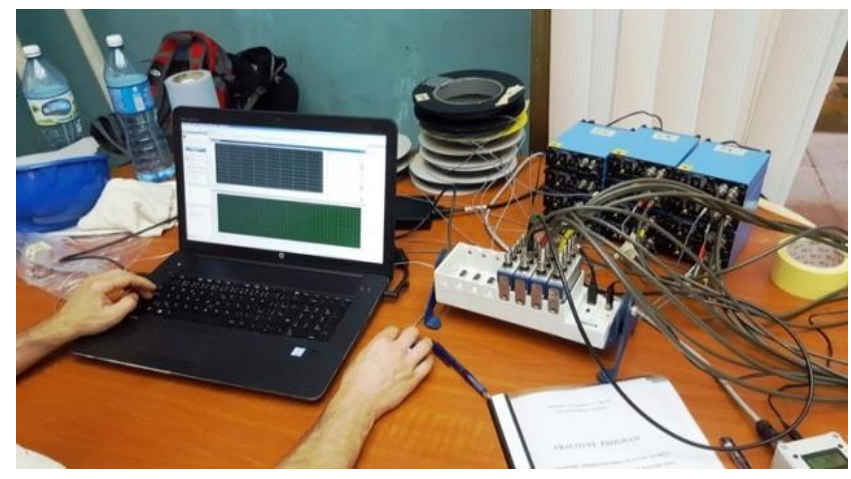

Fig. 5. Measuring station MS1 - UZ with NI software and hardware facilities.

The recorded signals from experimental tests were analysed by on-line methods in laboratory conditions (Fig. 6) or partly in situ using amplitude, frequency and amplitudephase analysis, by the method of spectral or correlation analysis. The TGFS-6 structure vibrations frequencies were obtained using spectral analysis method of recorded vibration amplitude time histories (TH) via accelerometers, which were considered as ergodic and stationary [2]. Spectral analysis calculation results (spectra, cross spectra $-\boldsymbol{S}_{\boldsymbol{k} k}(f), \boldsymbol{S}_{i k}(f)$; power spectral densities - PSD-Gii $(f)$; cross power spectral densities - CPSD- $\boldsymbol{G}_{i k}(f)$; coherence function - $\mathrm{CF}-\boldsymbol{\gamma}^{2} i k(\mathrm{f})$; phase function - PHA- $\boldsymbol{\Theta}_{i k}(f)$, etc.) were performed via National Instrument software package NI LabVIEV and partly by Modal Analysis (LabVIEV MA) and PC MO-AL. Fully application of the PC modal software packages was not possible utilized because of presence irrelevant vibration signals with high energy due to performance of others adjacent turbo-generators. Natural frequencies $\boldsymbol{f}_{\boldsymbol{b}} \boldsymbol{i}$ have been obtained by using the spectral analysis from recorded amplitude time histories $\boldsymbol{u}(t), \boldsymbol{v}(t)$, and $\boldsymbol{w}(t)$, due to various series of TGFS dynamic forced loading. As an example of the spectral analysis results are depictured on Figs.7, 8, 9. The results of the spectral analysis are fully described in [1]. 


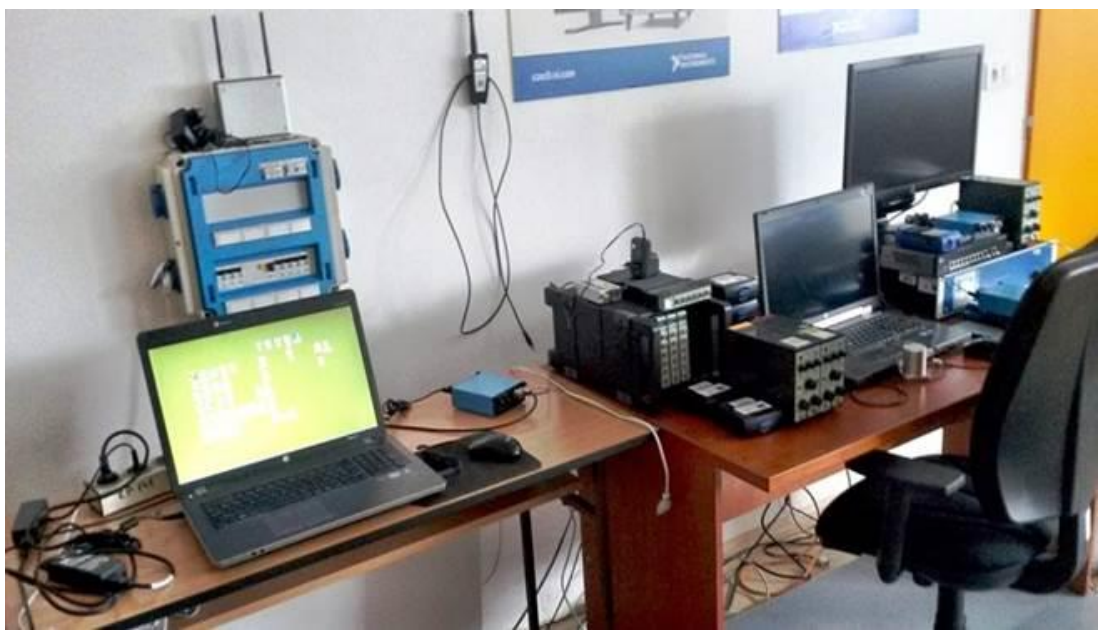

Fig. 6. Laboratory ZU AVL1 - view of data evaluation section.
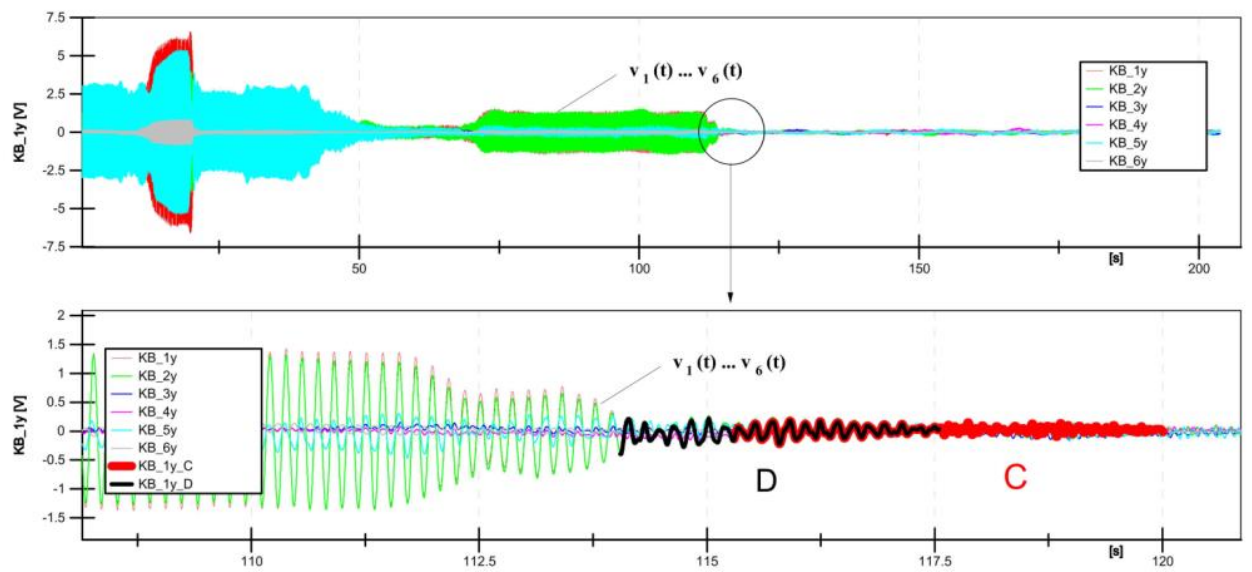

Fig. 7. Velocities amplitude vibration time history of $v_{i}(t)$ example; Test: S2Y-MB1 6.

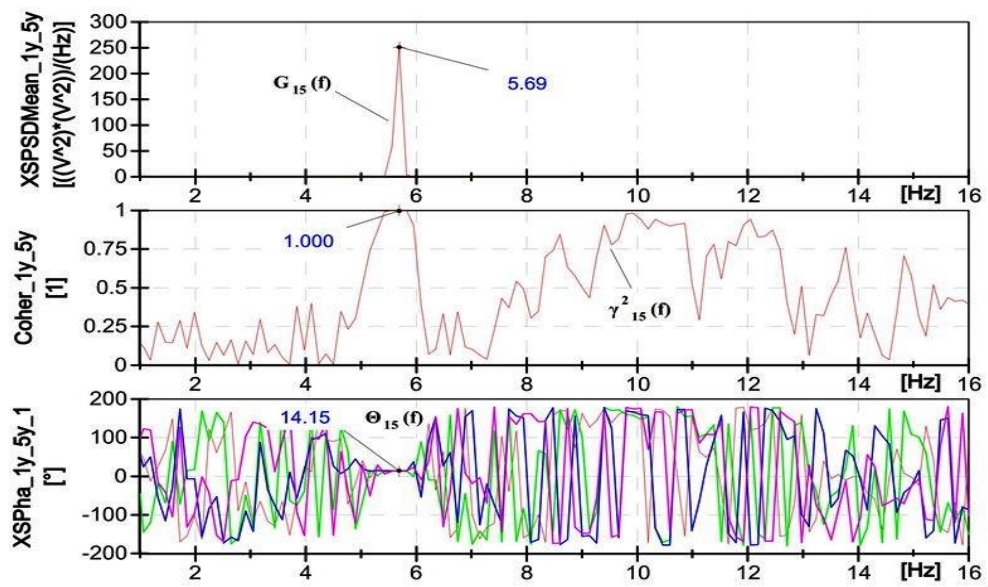

Fig. 8. Example of spectral analysis results: (a) CPSD $-\boldsymbol{G}_{15}(\mathrm{f})$; (b) $\mathrm{CF}-\boldsymbol{\gamma}^{2}{ }_{15}(\mathrm{f})$; (c) $\mathrm{PHA}-\boldsymbol{\Theta}_{15}(f)$. Test S2Y-MB1-3. 

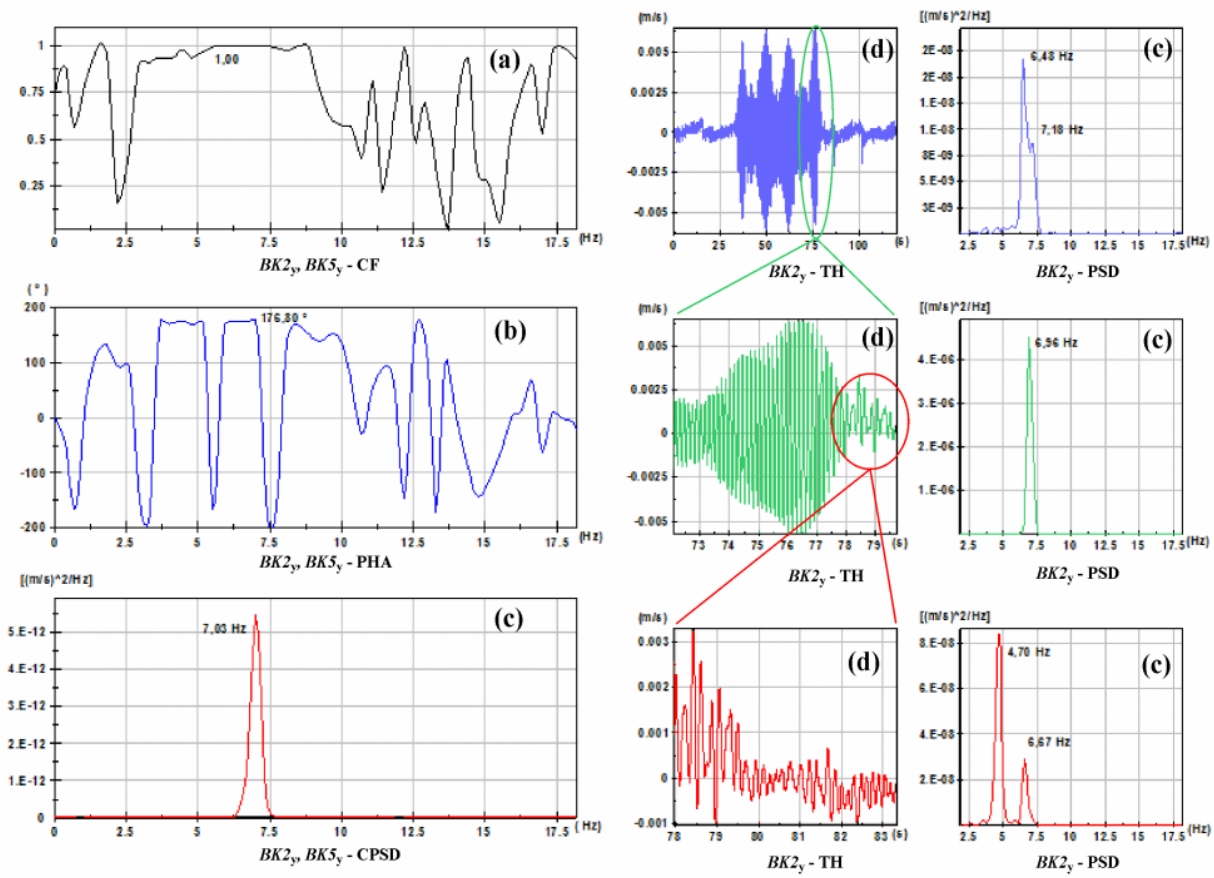

Fig. 9. Example of spectral analysis results: (a)CF- $\boldsymbol{\gamma}^{2} 25(f) ;(\mathbf{b}) \mathrm{PHA}-\boldsymbol{\Theta}_{25}(f) ;(\mathbf{c}) \mathrm{CPSD}-\boldsymbol{G}_{25}(f)$; (d) $\mathrm{TH}-\boldsymbol{v}_{2}(t)$.

\section{Conclusions}

- The dynamic analytical and experimental analysis method were a good tool for verification of foundation structure of the turbo-generator TG - 06 dynamic behaviour after the more than fifty years turbine performance and before it is put into operation a new turbo-generator.

- The comparison of the analytical and experimental analysis results enabled optimization of the TG foundation structure calculation FE model (FEM2) and determining the actual dynamic parameters of the existing TG fundament structure.

- The results of the experimental analysis also point out on the differences between measured and calculated TG structure natural frequencies values which finally make a decision on re-construction and strengthening of fundament structure.

- Finally, it enabled specifying the real behaviour of the TGFS - 6 in future with the possibility to determine of structure failures or operation life during the next turbogenerator service time.

- The utilization of the dynamic analysis method for the investigations of Industrial Structures is necessary to be more investigated to avoid possible extensive damages of TG and their foundation structures, $[5,6]$.

- A key ingredient to the successful foundation design for a turbo-generator is the careful engineering analysis of the foundation response to the dynamic loads from the anticipated operation of the machine, $[7,8,9,10,11,12]$. 
The research was supported by the Cultural and Educational Grant Agency of the Ministry of

Education, Science, Research and Sport of the Slovak Republic under the project No. 046ŽU-4/2018.

\section{References}

1. J. Bencat, et al., Technical Report No. P-140-1/17, Dynamic Analysis of Turbogenerator Machine Foundation Structure (TGFS), University of Zilina, p.60, (2017). (in Slovak)

2. J.S. Bendat, and A.G. Piersol, Engineering Applications of Correlation and Spectral Analysis, Wiley \& Sons, NJ, (1993)

3. M. Tomko, Technical report, SES a. s. Tlmace, Project of modernization of TGFS -6 /1st.Part, Dynamic calculation of the TGFS-6 structure, (2016)

4. M. Tomko, Technical report, SES a. s. Tlmace, Project of modernization of TGFS -6 /2nd.Part, Dynamic calculation of the TGFS-6 structure, (2017)

5. J. Bencat, Modal Analysis of Foundation Structure TG 11 in EMO Mochovce, Structural Dynamics, Eurodyn, Swets \& Zeitlinger, Lisse, (2002)

6. J. Bencat, et al. Technical Report No. HZ-08-SvF-2010, Dynamic Analysis of Turbogenerator Machine Foundation Structure TG15-EVO1 Vojany, University of Zilina, p.58, (2010), (in Slovak).

7. A. Major, Vibration Analysis and Design of Foundations for Machines and Turbines, Collet's, Collet's Holdings Limited, London, (1962).

8. K. G. Bhatia, ISET Journal of Earthquake Technology, 45 (1-2) (2008).

9. ISO 1940-1 Mechanical Vibration-Balance Quality Requirements of Rigid Rotors-Part 1: Determination of Permissible Residual Unbalance, (1993).

10. Bowels, J. E., Foundation Analysis and Design, 5th Edition, McGraw-Hill, (1996).

11. Whitman, R.V., Soil-Structure Interaction; Seismic Design for Nuclear Power Plants, MIT, (1969).

12.Wolf, J. P., Dynamic Soil- Structure Interaction, Elsevier Science B.V., (1998). 